\title{
Thin film photonic structures based on metal nanocrystals periodically distributed in a dielectric host
}

\author{
A. Suarez-Garcia, R. del Coso, R. Serna, J. Solis, C. N. Afonso \\ Instituto de Optica, Serrano 121, CSIC, Madrid, Spain
}

Nanostructured materials formed by metal nanocrystals (NCs) embedded in a dielectric matrix show special optical properties in the spectral range close to the plasmon resonance wavelength. Some of the potential applications in integrated optoelectronic devices that have been suggested for these materials are related either to their linear optical properties (long-wavelength pass filters) or the non-linear optical ones (ultrafast optical switches). For these applications it is essential to control their optical spectral response in the vicinity of the surface plasmon resonance (SPR). The absorption of these materials is usually described in terms of the optical properties of both the NCs and the host material, and it also depends on the dimensions and shape of the NCs. However, the optical response in metaldielectric structures can be also controlled by producing periodic photonic structures, as it has been theoretically demonstrated for the case of metal thin layers $[1,2]$. Nevertheless experimental reports based on photonic structuring of NCs systems have been very limited.

In this work, we have produced nanostructured thin films with an absorption that has been varied as a function of the distribution of the NCs. The NCs have been distributed in layers whose periodicity has been modeled in order to design the required response. To achieve consistent results an experimental-modeling feed-back approach has been followed. The results show that the optical transmission of such a system can be varied from that of a nanocomposite material in which the metal NCs are uniformly distributed in the dielectric volume and thus exhibiting a standard absorption resonance to that of a photonic structure. Some of these configurations have been experimentally produced and the agreement between modeled and exp erimental results is excellent.

The films have been produced by pulsed laser deposition, by independent ablation of $\mathrm{Al}_{2} \mathrm{O}_{3}$ and $\mathrm{Cu}$ targets. The deposition conditions have been selected to produce 6 layers of ellipsoidal NCs having average dimensions in the film plane of $\sim 5 \mathrm{~nm}$ and an average height $\leq 4 \mathrm{~nm}$ in a film with a total thickness of $50 \mathrm{~nm}$. A reference specimen with the NCs layers being produced at the center of the film and equally separated by $\sim 7 \mathrm{~nm}$ thick amorphous aluminum oxide

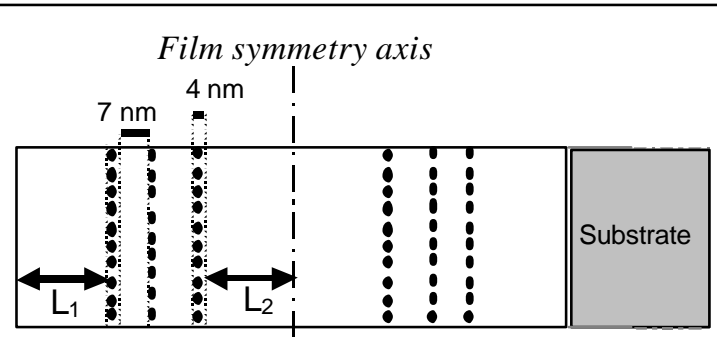

Figure 1. Schematic diagram of the nanostrucutred thin films. Dark dots represent $\mathrm{Cu}$ NCs distributed in six layers and white represents the amorphous $\mathrm{Al}_{2} \mathrm{O}_{3}$. $\mathrm{L}_{1}$ and $\mathrm{L}_{2}$ thicknesses of dielectric were changed as parameters in these samples, in order to obtain a photonic structure. The axis of symmetry of the thin film is represented by a discontinuous line. $\left(\mathrm{Al}_{2} \mathrm{O}_{3}\right)$ layers has first been produced and characterized. The film absorption spectrum shows a band centred at about $600 \mathrm{~nm}$ that has been attributed to the surface plasmon resonance, thus evidencing the presence of metal NCs in the film. The optical properties of such nanocomposite film have been analyzed by spectroscopic ellipsometry, assuming a multilayer structure $\left(\mathrm{Al}_{2} \mathrm{O}_{3} / \mathrm{Al}_{2} \mathrm{O}_{3}: \mathrm{Cu}\right.$ NCs). The analysis of the results allows us to determine the effective refractive index of the nanocomposite formed by the $\mathrm{Cu}$ NCs plus the $\mathrm{Al}_{2} \mathrm{O}_{3}$ that fills the space between them $\left(\mathrm{Al}_{2} \mathrm{O}_{3}: \mathrm{Cu}\right.$ $\mathrm{NCs}$ ). The optical constants of this nanocomposite material have been used to model and design photonic structures based on the scheme shown in Figure 1. Varying the values of $\mathrm{L}_{1}$ and $\mathrm{L}_{2}$ we have developed structures that allow an enhancement of the transmission up to $30 \%$ at the SPR wavelength in respect to the reference sample, while keeping the same $\mathrm{Cu}$ total content and the same $\mathrm{Cu}$ nanocrystal features. Films leading to the maximum and minimum transmission configurations have been synthesized by PLD, and the corresponding experimental absorption spectra show an excellent agreement with the simulated ones. The advantages of the different designs in terms of the optical response will be discussed.

\footnotetext{
${ }^{1}$ R. S. Benick, Y-K. Ion, R. W. Boyd, J.E. Sipe, Opt. Lett. 241416 (1999)

${ }^{2}$ T. Hattori, Jpn. J. Appl. Phys. 411349 (2002)
} 